\title{
Direct numerical solution of the Lippmann-Schwinger equation in coordinate space without partial-wave decomposition
}

\author{
Zeki C. Kuruoğlu* \\ Department of Chemistry, Bilkent University, 06800 Bilkent, Ankara, Turkey \\ (Received 8 April 2016; revised manuscript received 1 September 2016; published 7 November 2016)
}

\begin{abstract}
Direct numerical solution of the coordinate-space integral-equation version of the two-particle LippmannSchwinger (LS) equation is considered without invoking the traditional partial-wave decomposition. The singular kernel of the three-dimensional LS equation in coordinate space is regularized by a subtraction technique. The resulting nonsingular integral equation is then solved via the Nystrom method employing a direct-product quadrature rule for three variables. To reduce the computational burden of discretizing three variables, advantage is taken of the fact that, for central potentials, the azimuthal angle can be integrated out, leaving a two-variable reduced integral equation. A regularization method for the kernel of the two-variable integral equation is derived from the treatment of the singularity in the three-dimensional equation. A quadrature rule constructed as the direct product of single-variable quadrature rules for radial distance and polar angle is used to discretize the twovariable integral equation. These two- and three-variable methods are tested on the Hartree potential. The results show that the Nystrom method for the coordinate-space LS equation compares favorably in terms of its ease of implementation and effectiveness with the Nystrom method for the momentum-space version of the LS equation.
\end{abstract}

DOI: 10.1103/PhysRevE.94.053303

\section{INTRODUCTION}

Expansion in angular momentum states has hitherto been the usual ansatz for computational approaches to quantummechanical scattering problems. However, a critical reassessment of this strategy has occurred during recent years, especially for high-energy collisions [1] and within the context of few-body problems [2]. It has been realized that even for two-body problems involving central potentials, where the advantage due to the decoupling of partial-wave equations is manifest, the partial-wave expansion might lose its practical edge in high energies and for use in few-body calculations employing Faddeev-Yakubovski-type equations. Although the scattering amplitudes for most potentials are rather smooth, partial-wave amplitudes may show oscillatory behavior. Similarly, the off-shell two-body $T$ matrix usually has a simple structure, whereas partial-wave components might strongly oscillate. Under such circumstances, the partial-wave expansion involving an excessively large number of partial waves may be computationally impractical or even unreliable.

These observations suggest that, to treat two-particle scattering at high energies and within the context of few-particle dynamics, direct multivariable methods without recourse to expansions over angular momentum states might be more appropriate. Towards this end, multivariable methods has been investigated for the solution of the multivariable LippmannSchwinger (LS) equation in the momentum space [3-13]. For example, in Refs. [11,12] we have considered multivariable implementations of the Schwinger variational and Bateman methods for the two-body LS equation in momentum space. Significant progress has also been reported on the formal and computational aspects of solving the three-particle momentum-space Faddeev equations directly as five-variable

*kuruoglu@bilkent.edu.tr problems without invoking angular momentum decomposition $[2,14,15]$.

Calculational schemes based on momentum-space LS equations dominate the literature for two-body scattering computations, as exemplified in [3,5-13]. The coordinatespace version of the LS equation has received relatively less attention as a computational vehicle, although the coordinate-space partial-wave LS equation has been employed in connection with various types of Schwinger variational methods $[16,17]$. A direct numerical solution of the threedimensional coordinate-space LS equation for the transition operator is lacking. Presumably this is due to the singularity of the free Green's function $G_{0}\left(\mathbf{r}, \mathbf{r}^{\prime}\right)$ in the kernel of the LS equation. Similar singularities also occur in integral-equation formulations of electromagnetic scattering. In this article, a subtraction technique commonly used in computational electromagnetics $[18,19]$ is adopted for the three-dimensional LS equation. The principle aim of the present paper is to show that this singularity subtraction scheme paves the way for a simple and straightforward application of the Nystrom method [20] to directly solve the three-dimensional coordinate-space LS equation. The ensuing computational scheme is tested on a model problem that had served as a testing ground for other direct multivariable approaches $[4,11,12]$ that avoid partial-wave decomposition.

Employing a direct-product quadrature rule with a quadrature mesh $\left\{\mathbf{r}_{\alpha}, \alpha=1,2, \ldots, N_{Q}\right\}$ for integration over the computational domain in coordinate space, the Nystrom method yields a system of $N_{Q}$ linear equations for the (mixed representation) matrix elements $\left\langle\mathbf{r}_{\alpha}|T(E+)| \mathbf{q}_{0}\right\rangle$, where $\left|\mathbf{q}_{0}\right\rangle$ is the initial state with relative momentum $\mathbf{q}_{0}$ and energy $E=$ $\left|\mathbf{q}_{0}\right|^{2} / 2 \mu$. The momentum-space representation $\left\langle\mathbf{q}|T(E+)| \mathbf{q}_{0}\right\rangle$ is then obtained from $\left\langle\mathbf{r}|T(E+)| \mathbf{q}_{0}\right\rangle$ by the same threedimensional quadrature used in the Nystrom method. As usual with direct-product approaches to multivariable problems, this scheme suffers from the burden of dimensionality, with $N_{Q}$ growing very fast. Fortunately, however, for central potentials, 
the number of variables can be reduced by one. Using the fact that $G_{0}\left(\mathbf{r}, \mathbf{r}^{\prime}\right)$, and $\left\langle\mathbf{r}|T(E+)| \mathbf{q}_{0}\right\rangle$ for central potentials, depend on azimuthal angles only via the cosine of the difference of the azimuthal angles of the vectors involved, the azimuthal-angle dependence in the LS equation can be eliminated. The resulting two-variable integral equation can be considered as the integral-equation counterpart of the two-variable differential equation that was solved in Ref. [4] using the finite-element method to avoid partial-wave expansion.

Integration over the azimuthal angle weakens the singularity of the original Green's function, but the new reduced kernel still requires careful handling. We show that the regularized nonsingular three-dimensional integral equation can be reduced to obtain a regularized two-variable equation that is in a form ready for the quadrature discretization. Constructing a direct-product quadrature scheme by using $N_{r}$ points in $r$ and $N_{\theta}$ points in $\theta$, the Nystrom solution of the reduced LS equation yields a linear system of $N_{r} N_{\theta}$ equations that can be solved routinely in commonly available computational platforms.

The plan of this article is as follows. In Sec. II we discuss the reduction of the three-dimensional LS equation into a twovariable integral equation. Section III discusses the subtraction scheme for the removal of the singularity from the kernels of the three-dimensional and reduced forms of the LS equation. In Sec. IV the details of the computational implementation and results of calculations for the model potential are presented. In Sec. V we summarize our conclusions.

\section{FORMAL CONSIDERATIONS}

We consider the two-particle scattering problem for a central interparticle potential. Working in the center-of-mass frame, the relative momentum states are denoted by $|\mathbf{q}\rangle$ and the relative position states by $|\mathbf{r}\rangle$, with the normalizations $\left\langle\mathbf{r} \mid \mathbf{r}^{\prime}\right\rangle=\delta\left(\mathbf{r}-\mathbf{r}^{\prime}\right\rangle,\left\langle\mathbf{q} \mid \mathbf{q}^{\prime}\right\rangle=\delta\left(\mathbf{q}-\mathbf{q}^{\prime}\right\rangle$, and $\langle\mathbf{r} \mid \mathbf{q}\rangle=$ $e^{i \mathbf{r} \cdot \mathbf{q}} /(2 \pi)^{3 / 2}$. Atomic units will be used throughout this article.

The basic equation for the description of two-particle scattering is the Lippman-Schwinger equation for the twoparticle transition operator $T(z)$,

$$
T(z)=V+V G_{0}(z) T(z),
$$

where $V$ is the interaction potential between two particles and $G_{0}=\left(z-H_{0}\right)^{-1}$, with $z$ the (complex) energy of the two-particle system. For on-shell scattering, $z=E+i 0$ with $E=q_{0}^{2} / 2 \mu$, where $\mu$ is the reduced mass. Using a mixed representation, the matrix elements $T\left(\mathbf{r}, \mathbf{q}_{0}\right)\left[\equiv\left\langle\mathbf{r}|T(E+i 0)| \mathbf{q}_{0}\right\rangle\right]$ satisfy the three-dimensional integral equation

$$
T\left(\mathbf{r}, \mathbf{q}_{0}\right)=V(r)\left\langle\mathbf{r} \mid \mathbf{q}_{0}\right\rangle+V(r) \int d \mathbf{r}^{\prime} G_{0}\left(\mathbf{r}, \mathbf{r}^{\prime}\right) T\left(\mathbf{r}^{\prime}, \mathbf{q}_{0}\right),
$$

where $G_{0}\left(\mathbf{r}, \mathbf{r}^{\prime}\right)$ is the free Green's function, viz.,

$$
G_{0}\left(\mathbf{r}, \mathbf{r}^{\prime}\right)=\left\langle\mathbf{r}\left|G_{0}(E+i 0)\right| \mathbf{r}^{\prime}\right\rangle=-\frac{\mu}{2 \pi} \frac{e^{i q_{0}\left|\mathbf{r}-\mathbf{r}^{\prime}\right|}}{\left|\mathbf{r}-\mathbf{r}^{\prime}\right|} .
$$

Note that $T\left(\mathbf{r}, \mathbf{q}_{0}\right)$ is closely related to the scattering wave function $\psi_{\mathbf{q}_{0}}(\mathbf{r})$, viz.,

$$
T\left(\mathbf{r}, \mathbf{q}_{0}\right)=V(r) \psi_{\mathbf{q}_{0}}(\mathbf{r})
$$

where $\psi_{\mathbf{q}_{0}}$ is the solution of the LS equation for the wave function

$$
\psi_{\mathbf{q}_{0}}(\mathbf{r})=\left\langle\mathbf{r} \mid \mathbf{q}_{0}\right\rangle+\int d \mathbf{r}^{\prime} G_{0}\left(\mathbf{r}, \mathbf{r}^{\prime}\right) V\left(r^{\prime}\right) \psi_{\mathbf{q}_{0}}\left(\mathbf{r}^{\prime}\right) .
$$

Since $V(r)$ vanishes as $r$ gets sufficiently large, solving Eq. (2) for the amplitude $T\left(\mathbf{r}, \mathbf{q}_{0}\right)$ proves to be much more convenient computationally than directly working with Eq. (5) for the wave function. The momentum-space matrix elements $T\left(\mathbf{q}, \mathbf{q}_{0}\right)\left[\equiv\left\langle\mathbf{q}|T(E+i 0)| \mathbf{q}_{0}\right\rangle\right]$ of the transition operator can be calculated from the solution of Eq. (2) via a quadrature

$$
T\left(\mathbf{q}, \mathbf{q}_{0}\right)=\int d \mathbf{r}\langle\mathbf{q} \mid \mathbf{r}\rangle T\left(\mathbf{r}, \mathbf{q}_{0}\right) .
$$

We will denote the polar and azimuthal angles of the position vector $\mathbf{r}$ by $\theta$ and $\phi$ and those of the momentum vector $\mathbf{q}$ by $\theta_{q}$ and $\phi_{q}$, respectively. We will also use the notation $x$ for $\cos \theta, s$ for $\sin \theta, x_{q}$ for $\cos \theta_{q}$, and $s_{q}$ for $\sin \theta_{q}$. Since $\left|\mathbf{r}-\mathbf{r}^{\prime}\right|=\sqrt{r^{2}+r^{\prime 2}-2 r r^{\prime} x_{r r^{\prime}}}$ with $x_{r r^{\prime}}=x x^{\prime}+s s^{\prime} \cos (\phi-$ $\left.\phi^{\prime}\right)$, the dependence of $G_{0}\left(\mathbf{r}, \mathbf{r}^{\prime}\right)$ on azimuthal angles is only through the difference $\phi-\phi^{\prime}$. Similarly, $T\left(\mathbf{r}, \mathbf{q}_{0}\right)$ for central potentials depends on $r, q_{0}$, and $x_{r q_{0}}$. Here $x_{r q_{0}}$ is the cosine of the angle between vectors $\mathbf{r}$ and $\mathbf{q}_{0}$, i.e., $x_{r q_{0}}=\hat{\mathbf{r}} \cdot \hat{\mathbf{q}}_{0}$.

As $G_{0}\left(\mathbf{r}, \mathbf{r}^{\prime}\right)$, and $T\left(\mathbf{r}, \mathbf{q}_{0}\right)$ for central potentials, depend on azimuthal angles only through the differences $\phi-\phi^{\prime}$ and $\phi-\phi_{q_{0}}$, respectively, the azimuthal angle dependence can be eliminated from the LS equation in much the same way as in the momentum-space LS equation [3,5-13]. For this purpose we introduce reduced matrix elements of $G_{0}$ and $T$ via

$$
\begin{aligned}
& \hat{G}_{0}\left(r, x ; r^{\prime}, x^{\prime}\right)=\int_{0}^{2 \pi} d \phi G_{0}\left(\mathbf{r}, \mathbf{r}^{\prime}\right)=\int_{0}^{2 \pi} d \phi^{\prime} G_{0}\left(\mathbf{r}, \mathbf{r}^{\prime}\right), \\
& \hat{T}\left(r, x ; q_{0}, x_{q_{0}}\right)=\int_{0}^{2 \pi} d \phi T\left(\mathbf{r}, \mathbf{q}_{0}\right)=\int_{0}^{2 \pi} d \phi_{q_{0}} T\left(\mathbf{r}, \mathbf{q}_{0}\right) .
\end{aligned}
$$

We observe that, e.g., in Eq. (7), the first integral on the righthand side is independent of the azimuthal angle $\phi^{\prime}$ of $\mathbf{r}^{\prime}$, while the second integral is independent of $\phi$. Note that operators, matrix elements, and other quantities associated with the twovariable representation will be distinguished from those of the three-dimensional representation by a caret over the symbol.

Integrating both sides of Eq. (2) over $\phi$ and $\phi_{q_{0}}$, interchanging the order of integration over $\phi$ and $\phi^{\prime}$, and then making use of Eqs. (7) and (8), we obtain the reduced two-variable LS equation

$$
\begin{aligned}
\hat{T}\left(r, x ; q_{0}, x_{0}\right)= & V(r)\left\langle r x \mid q_{0} x_{q_{0}}\right\rangle+V(r) \int_{0}^{\infty} r^{\prime 2} d r^{\prime} \\
& \times \int_{-1}^{1} d x^{\prime} \hat{G}_{0}\left(r, x ; r^{\prime}, x^{\prime}\right) \hat{T}\left(r^{\prime}, x^{\prime} ; q_{0}, x_{q_{0}}\right),
\end{aligned}
$$

where

$$
\left\langle r x \mid q_{0} x_{q_{0}}\right\rangle=\int_{0}^{2 \pi} d \phi\left\langle\mathbf{r} \mid \mathbf{q}_{0}\right\rangle=(2 \pi)^{-1 / 2} e^{i q_{0} r x x_{q_{0}}} J_{0}\left(q_{0} r s s_{q_{0}}\right),
$$


with $J_{0}$ denoting the zeroth-order Bessel function. We write Eq. (7) in operator form as

$$
\hat{T}=\hat{V}+\hat{V} \hat{G}_{0} \hat{T}
$$

which is to be understood as an operator equation in the space of two-variable functions (of $r$ and $x$ ). On the other hand, the reduced version of Eq. (5) reads

$$
\hat{T}\left(q, x_{q} ; q_{0}, x_{q_{0}}\right)=\int r^{2} d r \int_{-1}^{1} d x\left\langle q x_{q} \mid r x\right\rangle \hat{T}\left(r, x ; q_{0}, x_{q_{0}}\right) .
$$

As discussed in [11], for an initial momentum vector $\mathbf{q}_{0}$ along the $z$ axis and a general final momentum vector $\mathbf{q}$, the transition matrix element $\left\langle\mathbf{q}|T| q_{0} \hat{\mathbf{z}}\right\rangle$ is given by

$$
\left\langle\mathbf{q}|T| q_{0} \hat{\mathbf{z}}\right\rangle=(2 \pi)^{-1} \hat{T}\left(q, x_{q} ; q_{0}, 1\right) .
$$

\section{REGULARIZATION OF THE KERNEL AND THE NYSTROM METHOD}

The standard method for the numerical solution of nonsingular integral equations is the Nystrom method, in which the integrals are replaced by sums via suitable quadrature rules and the resulting equations are collocated at the quadrature points. However, the singular nature of $G_{0}\left(\mathbf{r}, \mathbf{r}^{\prime}\right)$ does not allow the direct application of the Nystrom method to Eq. (2) in the full three-dimensional approach or to Eq. (9) in the two-variable version. We first recast the integrand of Eq. (2) as a sum of nonsingular and singular parts by subtracting and adding an (analytically) integrable singular term

$$
\begin{aligned}
G_{0}\left(\mathbf{r}, \mathbf{r}^{\prime}\right) T\left(\mathbf{r}^{\prime}, \mathbf{q}_{0}\right)= & -\frac{\mu}{2 \pi}\left\{\frac{e^{i q_{0}\left|\mathbf{r}-\mathbf{r}^{\prime}\right|} T\left(\mathbf{r}^{\prime}, \mathbf{q}_{0}\right)}{\left|\mathbf{r}-\mathbf{r}^{\prime}\right|}-\frac{T\left(\mathbf{r}, \mathbf{q}_{0}\right)}{\left|\mathbf{r}-\mathbf{r}^{\prime}\right|}\right\} \\
& -\frac{\mu}{2 \pi} \frac{T\left(\mathbf{r}, \mathbf{q}_{0}\right)}{\left|\mathbf{r}-\mathbf{r}^{\prime}\right|},
\end{aligned}
$$

where the term within curly brackets is no longer singular as $\mathbf{r}^{\prime} \rightarrow \mathbf{r}$, while the last term is analytically integrable over $\mathbf{r}^{\prime}$ for fixed r. Using Eq. (13) in Eq. (2), we obtain

$$
\begin{aligned}
T\left(\mathbf{r}, \mathbf{q}_{0}\right)= & V(r)\left\langle\mathbf{r} \mid \mathbf{q}_{0}\right\rangle-\frac{\mu}{2 \pi} V(r) I_{e}(\mathbf{r}) T\left(\mathbf{r}, \mathbf{q}_{0}\right) \\
& -\frac{\mu}{2 \pi} V(r) \int d \mathbf{r}^{\prime} \frac{e^{i q_{0}\left|\mathbf{r}-\mathbf{r}^{\prime}\right|} T\left(\mathbf{r}^{\prime}, \mathbf{q}_{0}\right)-T\left(\mathbf{r}, \mathbf{q}_{0}\right)}{\left|\mathbf{r}-\mathbf{r}^{\prime}\right|},
\end{aligned}
$$

where

$$
I_{e}(\mathbf{r})=\int d \mathbf{r}^{\prime} \frac{1}{\left|\mathbf{r}-\mathbf{r}^{\prime}\right|} .
$$

Two points must be made about this splitting. (i) Provided that, as $\mathbf{r} \rightarrow \mathbf{r}^{\prime},\left[e^{i q_{0}\left|\mathbf{r}-\mathbf{r}^{\prime}\right|} T\left(\mathbf{r}^{\prime}, \mathbf{q}_{0}\right)-T\left(\mathbf{r}, \mathbf{q}_{0}\right)\right]$ goes to zero faster than $\left|\mathbf{r}-\mathbf{r}^{\prime}\right|$ does, the subtracted kernel in Eq. (14) is nonsingular and the integral over $\mathbf{r}^{\prime}$ in Eq. (14) can now be approximated by a quadrature rule. (ii) The term $I_{e}(\mathbf{r})$ diverges as $|\mathbf{r}| \rightarrow \infty$, but it occurs in Eq. (14) multiplied by $T\left(\mathbf{r}, \mathbf{q}_{0}\right)$ and $V(r)$, both of which vanish in the same limit. In the practical implementation of Eq. (14), the computational domain for $\mathbf{r}$ is truncated by introducing a cutoff $r_{\max }$ for the radial variable $r$.
We then have

$$
\begin{aligned}
I_{e}(\mathbf{r}) & =\int_{0}^{r_{\max }} r^{\prime 2} d r^{\prime} \int_{-1}^{+1} d x^{\prime} \int_{0}^{2 \pi} d \phi^{\prime}\left|\mathbf{r}-\mathbf{r}^{\prime}\right|^{-1} \\
& =4 \pi\left(\frac{r_{\max }^{2}}{2}-\frac{r^{2}}{6}\right),
\end{aligned}
$$

where $r<r_{\max }$. The need to introduce this cutoff might limit the applicability of the method to short-range potentials. Note that $I_{e}(\mathbf{r})$ is in fact independent of the orientation of $\mathbf{r}$. The same integral $\int_{0}^{r_{\max }} r^{\prime 2} d r^{\prime} \int_{-1}^{+1} d x^{\prime} \int_{0}^{2 \pi} d \phi^{\prime}\left|\mathbf{r}-\mathbf{r}^{\prime}\right|^{-1}$ also occurs as part of the second term on the right-hand side of Eq. (14), where, however, it is to be evaluated via the quadrature rule chosen for the discretization of the integral equation.

Let $\mathbf{r}_{\alpha}, \alpha=1,2, \ldots, N_{Q}$, be a set of quadrature points over the computational $\mathbf{r}$ domain with corresponding weights denoted by $w_{\alpha}$. We will construct this three-dimensional quadrature rule as the direct product of single-variable quadrature rules for $r, x$, and $\phi$. Let $\left\{r_{i}, i=1, \ldots, N_{r}\right\}$, $\left\{x_{j}, j=1, \ldots, N_{x}\right\}$, and $\left\{\phi_{k}, k=1, \ldots, N_{\phi}\right\}$ be the sets of quadrature points chosen for $r, x$, and $\phi$ over the intervals $\left[0, r_{\max }\right],[-1,1]$, and $[0,2 \pi]$, with corresponding weights $\left\{w_{r_{i}}\right\},\left\{w_{x_{j}}\right\}$, and $\left\{w_{\phi_{k}}\right\}$, respectively. Using the composite index $\alpha$ for the index combination $(i j k)$, the position vector whose spherical components are $r_{i}, x_{j}$, and $\phi_{k}$ is denoted by $\mathbf{r}_{\alpha}, \alpha=1,2, \ldots, N_{Q}$, where $N_{Q}=N_{r} N_{x} N_{\phi}$. With this notation, the weights of the three-dimensional quadrature rule are $w_{\alpha}=r_{i}^{2} w_{r_{i}} w_{x_{j}} w_{\phi_{k}}$.

Approximating the integral over $\mathbf{r}^{\prime}$ in Eq. (14) by the quadrature rule chosen and collocating $\mathbf{r}$ at the quadrature points, we obtain

$$
\begin{aligned}
T\left(\mathbf{r}_{\alpha}, \mathbf{q}_{0}\right)= & V\left(\left|\mathbf{r}_{\alpha}\right|\right)\left\langle\mathbf{r}_{\alpha} \mid \mathbf{q}_{0}\right\rangle+V\left(\left|\mathbf{r}_{\alpha}\right|\right) C\left(\mathbf{r}_{\alpha}\right) T\left(\mathbf{r}_{\alpha}, \mathbf{q}_{0}\right) \\
& +V\left(\left|\mathbf{r}_{\alpha}\right|\right) \sum_{\alpha^{\prime}=1}^{N_{Q}} \bar{\delta}_{\alpha \alpha^{\prime}} G_{0}\left(\mathbf{r}_{\alpha}, \mathbf{r}_{\alpha^{\prime}}\right) w_{\alpha^{\prime}} T\left(\mathbf{r}_{\alpha^{\prime}}, \mathbf{q}_{0}\right),
\end{aligned}
$$

where $\alpha=1,2, \ldots, N_{Q}, \bar{\delta}_{\alpha \alpha^{\prime}}=1-\delta_{\alpha \alpha^{\prime}}$, and

$$
C\left(\mathbf{r}_{\alpha}\right)=-\frac{\mu}{2 \pi}\left[I_{e}\left(\mathbf{r}_{\alpha}\right)-I_{a}\left(\mathbf{r}_{\alpha}\right)\right]
$$

Here $I_{e}$ is as defined in Eq. (16) and

$$
I_{a}\left(\mathbf{r}_{\alpha}\right)=\sum_{\alpha^{\prime}=1}^{N_{Q}} \bar{\delta}_{\alpha \alpha^{\prime}} w_{\alpha^{\prime}} /\left|\mathbf{r}_{\alpha}-\mathbf{r}_{\alpha^{\prime}}\right|
$$

For a given initial momentum vector $\mathbf{q}_{0}$, Eq. (17) represents a system of $N_{Q}$ linear equations.

Although the integration over $\phi$ and/or $\phi^{\prime}$ implicit in the definition of $\hat{G}_{0}\left(r, x ; r^{\prime}, x^{\prime}\right)$ weakens the singularity of the Green's function $G_{0}\left(\mathbf{r}, \mathbf{r}^{\prime}\right)$, the numerical treatment of the two-variable LS equation also requires a careful handling of the kernel. Application to Eq. (9) of the same subtraction trick leads to

$$
\begin{aligned}
\hat{T}\left(r, x ; q_{0}, x_{0}\right)= & V(r)\left\langle r x \mid q_{0} x_{q_{0}}\right\rangle \\
& -(\mu / 2 \pi) V(r) I_{e}(r) T\left(r, x ; q_{0}, x_{0}\right) \\
& +V(r) \int_{0}^{\infty} r^{\prime 2} d r^{\prime} \int_{-1}^{1} d x^{\prime}
\end{aligned}
$$




$$
\begin{aligned}
& \times \int d \phi^{\prime}\left\{G_{0}\left(\mathbf{r}, \mathbf{r}^{\prime}\right) T\left(r^{\prime}, x^{\prime} ; q_{0}, x_{0}\right)\right. \\
& \left.+(\mu / 2 \pi)\left|\mathbf{r}-\mathbf{r}^{\prime}\right|^{-1} T\left(r, x ; q_{0}, x_{0}\right)\right\} .
\end{aligned}
$$

In Eq. (20), the azimuthal angle $\phi$ of the vector $\mathbf{r}$ has been set to zero. This choice can be made because the integrals $\int d \phi^{\prime} G_{0}\left(\mathbf{r}, \mathbf{r}^{\prime}\right)$ and $\int d \phi^{\prime}\left|\mathbf{r}-\mathbf{r}^{\prime}\right|^{-1}$ are independent of the azimuthal angle of $\mathbf{r}$, as pointed out in connection with Eq. (7).

Approximating the integrals over $r^{\prime}, x^{\prime}$, and $\phi^{\prime}$ in Eq. (20) by the quadrature rule and collocating $r$ and $x$ at the quadrature points $\left\{r_{i}, i=1,2, \ldots, N_{r}\right\}$ and $\left\{x_{j}, j=1,2, \ldots, N_{x}\right\}$, respectively, we obtain a system of $N_{r} N_{x}$ equations

$$
\begin{aligned}
\hat{T}\left(r_{i}, x_{j} ; q_{0}, x_{0}\right)= & V\left(r_{i}\right)\left\langle r_{i} x_{j} \mid q_{0} x_{q_{0}}\right\rangle \\
& -V\left(r_{i}\right) \hat{C}\left(r_{i}, x_{j}\right) \hat{T}\left(r_{i}, x_{j} ; q_{0}, x_{q_{0}}\right) \\
& +V\left(r_{i}\right) \sum_{i^{\prime}=1}^{N_{r}} \sum_{j^{\prime}=1}^{N_{x}} \hat{G}_{0}\left(r_{i}, x_{j} ; r_{i^{\prime}}, x_{j^{\prime}}\right) r_{i^{\prime}}^{2} w_{r_{i^{\prime}}} w_{x_{j^{\prime}}} \\
& \times \hat{T}\left(r_{i^{\prime}}, x_{j^{\prime}} ; q_{0}, x_{q_{0}}\right),
\end{aligned}
$$

where

$$
\begin{aligned}
\hat{G}_{0}\left(r_{i}, x_{j} ; r_{i^{\prime}}, x_{j^{\prime}}\right) & =-(\mu / 2 \pi) \sum_{k=1}^{N_{\phi}} w_{\phi_{k}} e^{i q_{0} d\left(i j, i^{\prime} j^{\prime} ; k\right)} / d\left(i j, i^{\prime} j^{\prime} ; k\right), \\
d\left(i j, i^{\prime} j^{\prime} ; k\right) & =\left[r_{i}^{2}+r_{i^{\prime}}^{2}-2 r_{i} r_{i^{\prime}}\left(x_{j} x_{j^{\prime}}+s_{j} s_{j^{\prime}} \cos \phi_{k}\right)\right]^{1 / 2}, \\
\hat{C}\left(r_{i}, x_{j}\right) & =-(\mu / 2 \pi)\left[I_{e}\left(r_{i}\right)-I_{a}\left(r_{i}, x_{j}\right)\right], \\
I_{a}\left(r_{i}, x_{j}\right) & =\sum_{k=1}^{N_{\phi}} w_{\phi_{k}} / d\left(i j, i^{\prime} j^{\prime} ; k\right) .
\end{aligned}
$$

The singularity-correction term $\hat{C}\left(r_{i}, x_{j}\right)$ turns out to be crucial for the success of the Nystrom method.

Computational implementations of Eqs. (17) and (21) for $T\left(r_{i}, x_{j}, \phi ; q_{0}, x_{0}, \phi_{0}\right)$ and $\hat{T}\left(r_{i}, x_{j} ; q_{0}, x_{0}\right)$, respectively, involve complex arithmetic. To avoid complex arithmetic and also to reduce memory and CPU requirements, it is possible to organize the computations in terms of the real $K$-matrix elements $K\left(r_{i}, x_{j}, \phi_{k} ; q_{0}, x_{0}, \phi_{0}\right)$ and $\hat{K}\left(r_{i}, x_{j} ; q_{0}, x_{0}\right)$. The $K$ matrix versions of Eqs. (17) and (21) are obtained by replacing $G_{0}\left(\mathbf{r}, \mathbf{r}^{\prime}\right)$ by its real part $G_{0}^{P}\left(\mathbf{r}, \mathbf{r}^{\prime}\right)=\cos q_{0}\left|\mathbf{r}-\mathbf{r}^{\prime}\right| /\left|\mathbf{r}-\mathbf{r}^{\prime}\right|$. After solving for $K\left(r, x, \phi ; q_{0}, x^{\prime}, \phi^{\prime}\right)$ and $\hat{K}\left(r, x ; q_{0} x^{\prime}\right)$, the momentum-space $K$-matrix elements $K\left(q_{0}, x, \phi ; q_{0}, x^{\prime}, \phi^{\prime}\right)$ and $\hat{K}\left(q_{0}, x ; q_{0}, x^{\prime}\right)$ are constructed by evaluating the $K$-matrix versions of Eqs. (6) and (12) via the same quadrature rule used in the Nystrom method. To obtain the $T$-matrix elements, the Heitler damping equation $T=K-i \pi K \delta\left(E-H_{0}\right) T$ has to be solved. In the three-dimensional case, this is an integral equation in two angular variables and after discretization of the angular variables reads

$$
\begin{aligned}
& T\left(q_{0}, x_{j}, \phi_{k} ; q_{0}, x_{0}, \phi_{0}\right) \\
& =\quad K\left(q_{0}, x_{j}, \phi_{k} ; q_{0}, x_{0}, \phi_{0}\right)-\left(i \pi \mu q_{0}\right) \\
& \quad \times \sum_{j^{\prime}=1}^{N_{x}} \sum_{k^{\prime}=1}^{N_{\phi}} w_{x_{j^{\prime}}} w_{\phi_{k^{\prime}}} K\left(q_{0}, x_{j}, \phi_{k} ; q_{0}, x_{j^{\prime}}, \phi_{k^{\prime}}\right) \\
& \quad \times T\left(q_{0}, x_{j^{\prime}}, \phi_{k^{\prime}} ; q_{0}, x_{0}, \phi_{0}\right)
\end{aligned}
$$

which represents a system of $N_{x} N_{\phi}$ linear equations. For the two-variable case, the discretized Heitler equation reads

$$
\begin{aligned}
\hat{T}\left(q_{0}, x_{j} ; q_{0}, x_{0}\right)= & \hat{K}\left(q_{0}, x_{j} ; q_{0}, x_{0}\right) \\
& -\left(i \pi \mu q_{0}\right) \sum_{j^{\prime}=1}^{N_{x}} w_{x_{j^{\prime}}} \hat{K}\left(q_{0}, x_{j} ; q_{0}, x_{j^{\prime}}\right) \\
& \times \hat{T}\left(q_{0}, x_{j^{\prime}} ; q_{0}, x_{0}\right),
\end{aligned}
$$

representing a system of $N_{x}$ linear equations. If we invoke the Born approximation for the $K$ matrix, i.e., set $\hat{K}\left(q_{0}, x_{j} ; q_{0}, x_{j^{\prime}}\right)=\hat{V}\left(q_{0}, x_{j} ; q_{0}, x_{j^{\prime}}\right)$, in the above equation, the resulting $T$ matrix will be referred to as the $K$ Born approximation.

\section{COMPUTATIONAL IMPLEMENTATION AND RESULTS}

The two- and three-variable implementations of the Nystrom method discussed in the previous section are tested on the Hartree potential

$$
V(r)=V_{0} e^{-\lambda r}\left(1+\frac{1}{r}\right) .
$$

This model was chosen because Shertzer and Temkin [4] had used it in a similar context, namely, to test a two-variable finiteelement approach for solving the (two-variable) Schrödinger equation without partial-wave expansion. The values used for the potential parameters are adopted from Ref. [4], i.e., $V_{0}=-2.0$ and $\lambda=2.0$, and the reduced mass $\mu=0.5$.

Problems with larger reduced masses and high collision energies are expected to involve a larger number of partial waves and therefore provide a context more conducive to the direct (non-partial-wave) multivariable approaches such as the present one. Thus, in order to compare the computational performance of the present direct multivariable Nystrom method in coordinate space with that of a partial-wave approach (namely, the Nystrom method for the partial-wave LS equation in momentum space), a variant of the model with reduced mass $\mu=2.0$ is also considered.

Reference results for the Hartree potential were obtained with momentum-space Nystrom calculations, as reported in Ref. [11], and are stable within seven digits after the decimal point to further variations in computational parameters. The calculations of Tables VI and VII aimed at finding the number $j_{\text {max }}$ of partial waves to be retained for convergence in the partial-wave decomposition were carried out by solving partial-wave LS equations in momentum space via the singlevariable Nystrom scheme, separately for each partial wave $j$, with $j=0,1, \ldots, j_{\max }$.

The cutoff $r_{\max }$ for the variable $r$ is taken as 12, although a value of 8 is sufficient for about four to five digit accuracy. The quadrature grid for $r$ is uneven: denser for small $r$, coarser for large $r$. The interval $\left[0, r_{\max }\right]$ is divided into four subintervals, $(0,0.5),(0.5,2),(2,8)$, and $(8,12)$, which are in turn subdivided into $I_{i}$ elements, $i=1,2,3,4$. Each element is mapped to $[-1,1]$, and a set of $n_{r}$ Gauss-Legendre points and their corresponding weights are generated for each element. By combining the quadrature points and weights for all elements, a composite quadrature rule of $N_{r}$ points is generated. Here $N_{r}=I_{r} n_{r}$, with $I_{r}\left(\equiv I_{1}+I_{2}+I_{3}+I_{4}\right)$ denoting the total 
TABLE I. Convergence study for the three-dimensional Nystrom method with respect to the number of quadrature points $N_{r}$ for the variable $r$. Listed are the scattering amplitudes $A(x ; E)$ at $E=0.25$ and $E=4.0$ obtained with different values of $N_{r}$. The numbers of quadrature points used for the angular variables are $N_{x}=20$ and $N_{\phi}=20$.

\begin{tabular}{|c|c|c|c|c|c|c|}
\hline \multirow[b]{2}{*}{$N_{r}$} & \multicolumn{3}{|c|}{$\operatorname{Re} A(x ; E)$} & \multicolumn{3}{|c|}{$\operatorname{Im} A(x ; E)$} \\
\hline & $x=1.0$ & $x=0.0$ & $x=-1.0$ & $x=1.0$ & $x=0.0$ & $x=-1.0$ \\
\hline & & & $E=0.25$ & & & \\
\hline 64 & 1.04010 & 0.86194 & 0.72560 & 1.49979 & 1.49571 & 1.49169 \\
\hline 72 & 1.04009 & 0.86194 & 0.72560 & 1.49964 & 1.49554 & 1.49153 \\
\hline 80 & 1.04008 & 0.86192 & 0.72558 & 1.49957 & 1.49549 & 1.49146 \\
\hline 88 & 1.04008 & 0.86192 & 0.72558 & 1.49957 & 1.49549 & 1.49146 \\
\hline 100 & 1.04006 & 0.86190 & 0.72556 & 1.49953 & 1.49545 & 1.49142 \\
\hline \multirow[t]{2}{*}{ Ref. [11] } & 1.03980 & 0.86163 & 0.72529 & 1.49968 & 1.49559 & 1.49155 \\
\hline & & & $E=4.0$ & & & \\
\hline 64 & 0.97876 & 0.16262 & 0.05086 & 0.29889 & 0.19713 & 0.14466 \\
\hline 72 & 0.97871 & 0.16259 & 0.05086 & 0.29893 & 0.19715 & 0.14462 \\
\hline 80 & 0.97868 & 0.16258 & 0.05085 & 0.29896 & 0.19714 & 0.14459 \\
\hline 88 & 0.97868 & 0.16258 & 0.05085 & 0.29897 & 0.19714 & 0.14459 \\
\hline 100 & 0.97866 & 0.16257 & 0.05085 & 0.29899 & 0.19713 & 0.14458 \\
\hline Ref. [11] & 0.97860 & 0.16253 & 0.05083 & 0.29917 & 0.19715 & 0.14454 \\
\hline
\end{tabular}

number of elements for $r$. Similarly, the interval $[-1,1]$ for the variable $x$ is divided into $I_{x}$ equal elements, with $n_{x}$ Gauss-Legendre points chosen in each element. Thus, a composite quadrature rule with $N_{x}=I_{x} n_{x}$ is generated. For the $\phi$ integrals, the interval $[0,2 \pi]$ is divided into $I_{\phi}$ equal elements, with $n_{\phi}$ Gauss-Legendre points in each element, yielding a composite quadrature rule with $N_{\phi}=I_{\phi} n_{\phi}$.

\section{A. Results of three-dimensional calculations}

Tables I and II report the results of the Nystrom calculations with different values of $N_{r}, N_{x}$, and $N_{\phi}$ for $\mu=0.5$. Table I probes the convergence with respect to $N_{r}$ with fixed $N_{x}$ and $N_{\phi}$ and Table II with respect to $N_{x}$ and $N_{\phi}$ with fixed $N_{r}$. Shown are the real and imaginary parts of the scattering amplitude

$$
A(x ; E) \equiv-4 \pi^{2} \mu\left\langle q_{0} x \phi|T(E)| q_{0} x_{0} \phi_{0}\right\rangle
$$

for three values of $x$, with $x_{0}=1$ and $\phi_{0}=\phi=0$.
The largest calculation in Table I uses $N_{r}=100$ with the following distribution of quadrature points for $r: I_{1}=6, I_{2}=$ $6, I_{3}=9, I_{4}=4$, and $n_{r}=4$. With $N_{x}=20$ and $N_{\phi}=20$, this corresponds to $N_{Q}=40000$. The resulting system of equations is solved either by a direct out-of-core equation solver (a block-by-block scheme of Gaussian elimination with partial pivoting that was described earlier in [21]) or by Padé resummation of the Born series generated from Eq. (17). Typically, the $[8,7]$ approximant is sufficient for convergence. It is reassuring that direct and Padé solutions agree within at least eight significant digits for a given set of computational parameters.

The calculations presented in this article have been mostly done with complex arithmetic using the $T$-matrix equations. However, these results were verified by also carrying out $K$-matrix calculations. The results of the $T$ - and $K$-matrix schemes agree within at least eight significant figures. Although the $K$-matrix scheme is favorable in terms of memory and CPU demands, the need to solve the Heitler equation makes it a little more involved to implement.

TABLE II. Convergence study for the three-dimensional Nystrom method with respect to the number of quadrature points $N_{x}$ and $N_{\phi}$ for the variables $x$ and $\phi$, respectively. Listed are the scattering amplitudes $A(x ; E)$ at $E=0.25$ and $E=4.0$. For calculations of this table, $N_{r}=100$.

\begin{tabular}{|c|c|c|c|c|c|c|c|}
\hline \multirow[b]{2}{*}{$N_{x}$} & \multirow[b]{2}{*}{$N_{\phi}$} & \multicolumn{3}{|c|}{$\operatorname{Re} A(x ; E)$} & \multicolumn{3}{|c|}{$\operatorname{Im} A(x ; E)$} \\
\hline & & $x=1.0$ & $x=0.0$ & $x=-1.0$ & $x=1.0$ & $x=0.0$ & $x=-1.0$ \\
\hline & & & & $E=0.25$ & & & \\
\hline 12 & 12 & 1.04111 & 0.86299 & 0.72669 & 1.49811 & 1.49404 & 1.49002 \\
\hline 16 & 16 & 1.04032 & 0.86217 & 0.72669 & 1.49923 & 1.49515 & 1.49113 \\
\hline 20 & 20 & 1.04006 & 0.86190 & 0.72556 & 1.49953 & 1.49545 & 1.49142 \\
\hline \multicolumn{2}{|c|}{ Ref. [11] } & 1.03980 & 0.86163 & 0.72529 & 1.49968 & 1.49559 & 1.49155 \\
\hline & & & & $E=4.0$ & & & \\
\hline 12 & 12 & 0.97858 & 0.16202 & 0.05101 & 0.29853 & 0.19696 & 0.14456 \\
\hline 16 & 16 & 0.97866 & 0.16254 & 0.05087 & 0.29886 & 0.19710 & 0.14459 \\
\hline 20 & 20 & 0.97866 & 0.16257 & 0.05085 & 0.29899 & 0.19713 & 0.14458 \\
\hline \multicolumn{2}{|c|}{ Ref. [11] } & 0.97860 & 0.16253 & 0.05083 & 0.29917 & 0.19715 & 0.14454 \\
\hline
\end{tabular}


TABLE III. Convergence study for the two-variable Nystrom method with respect to the number of quadrature points $N_{r}$ for the variable $r$. Listed are the scattering amplitudes $A(x ; E)$ at $E=0.25$ and $E=4.0$ for different values of $N_{r}$. For calculations reported in this table, the number $N_{x}$ of quadrature points for the variable $x$ is 100 .

\begin{tabular}{|c|c|c|c|c|c|c|}
\hline \multirow[b]{2}{*}{$N_{r}$} & \multicolumn{3}{|c|}{$\operatorname{Re} A(x ; E)$} & \multicolumn{3}{|c|}{$\operatorname{Im} A(x ; E)$} \\
\hline & $x=1.0$ & $x=0.0$ & $x=-1.0$ & $x=1.0$ & $x=0.0$ & $x=-1.0$ \\
\hline \multicolumn{7}{|c|}{$E=0.25$} \\
\hline 72 & 1.0397587 & 0.8615963 & 0.7252521 & 1.4997458 & 1.4956522 & 1.4916166 \\
\hline 108 & 1.0397867 & 0.8616244 & 0.7252803 & 1.4998965 & 1.4956029 & 1.4915674 \\
\hline 144 & 1.0397918 & 0.8616296 & 0.7252855 & 1.4996874 & 1.4955938 & 1.4915583 \\
\hline 180 & 1.0397935 & 0.8616313 & 0.7252872 & 1.4996845 & 1.4955909 & 1.4915553 \\
\hline 216 & 1.0397940 & 0.8616317 & 0.7252876 & 1.4996837 & 1.4955901 & 1.4915545 \\
\hline 252 & 1.0397942 & 0.8616319 & 0.7252878 & 1.4996833 & 1.4955897 & 1.4915542 \\
\hline Ref. [11] & 1.0397948 & 0.8616326 & 0.7252885 & 1.4996821 & 1.4955886 & 1.4915530 \\
\hline \multicolumn{7}{|c|}{$E=4.0$} \\
\hline 72 & 0.9786075 & 0.1625322 & 0.0508286 & 0.2991803 & 0.1971639 & 0.1445539 \\
\hline 108 & 0.9786048 & 0.1625306 & 0.0508278 & 0.2991709 & 0.1971550 & 0.1445454 \\
\hline 144 & 0.9786043 & 0.1625304 & 0.0508276 & 0.2991692 & 0.1971533 & 0.1445438 \\
\hline 180 & 0.9786041 & 0.1625303 & 0.0508276 & 0.2991687 & 0.1971528 & 0.1445433 \\
\hline 216 & 0.9786041 & 0.1625302 & 0.0508276 & 0.2991685 & 0.1971527 & 0.1445432 \\
\hline 252 & 0.9786041 & 0.1625302 & 0.0508275 & 0.2991684 & 0.1971526 & 0.1445431 \\
\hline Ref. [11] & 0.9786040 & 0.1625302 & 0.0508275 & 0.2991682 & 0.1971524 & 0.1445429 \\
\hline
\end{tabular}

The accuracy of the results of the calculations in Table I is limited to three to four significant figures, because rather modest values for $N_{x}$ and $N_{\phi}$ were used to avoid excessively large $N_{Q}$. The largest absolute error for the scattering amplitudes reported in Table $I$ is less than $3 \times 10^{-4}$ for $E=0.25$ and less than $6 \times 10^{-5}$ for $E=4$. The two-dimensional calculations of the next section suggest that more realistic values for $N_{x}$ and $N_{\phi}$ must be on the order of 40-60 if we ask for results accurate to within five to six significant figures.

TABLE IV. Convergence of the Nystrom solution of the two-variable LS equation with respect to $N_{x}$, the number of quadrature points for the variable $x$. Listed are the scattering amplitudes $A(x ; E)$ at $E=0.25$ and $E=4.0$ for different values of $N_{x}$. For calculations reported in this table, the number $N_{r}$ of quadrature points for the variable $r$ is 360 .

\begin{tabular}{|c|c|c|c|c|c|c|}
\hline \multirow[b]{2}{*}{$N_{x}$} & \multicolumn{3}{|c|}{$\operatorname{Re} A(x ; E)$} & \multicolumn{3}{|c|}{$\operatorname{Im} A(x ; E)$} \\
\hline & $x=1.0$ & $x=0.0$ & $x=-1.0$ & $x=1.0$ & $x=0.0$ & $x=-1.0$ \\
\hline & & & $E=0.25$ & & & \\
\hline 8 & 1.0399658 & 0.8618044 & 0.7254623 & 1.4993804 & 1.4952869 & 1.4912515 \\
\hline 16 & 1.0398058 & 0.8616437 & 0.7252997 & 1.4996627 & 1.4955692 & 1.4915336 \\
\hline 24 & 1.0397965 & 0.8616343 & 0.7252902 & 1.4996792 & 1.4955857 & 1.4915501 \\
\hline 32 & 1.0397954 & 0.8616332 & 0.7252892 & 1.4996811 & 1.4955875 & 1.4915520 \\
\hline 40 & 1.0397946 & 0.8616324 & 0.7252884 & 1.4996825 & 1.4955889 & 1.4915533 \\
\hline 60 & 1.0397942 & 0.8616320 & 0.7252879 & 1.4996831 & 1.4955896 & 1.4915540 \\
\hline 80 & 1.0397942 & 0.8616319 & 0.7252878 & 1.4996833 & 1.4955897 & 1.4915542 \\
\hline 100 & 1.0397941 & 0.8616319 & 0.7252878 & 1.4996833 & 1.4955898 & 1.4915542 \\
\hline \multirow[t]{2}{*}{ Ref. [11] } & 1.0397948 & 0.8616326 & 0.7252885 & 1.4996821 & 1.4955886 & 1.4915530 \\
\hline & & & $E=4.0$ & & & \\
\hline 8 & 0.9787138 & 0.1623328 & 0.0427280 & 0.2991517 & 0.1971215 & 0.1445077 \\
\hline 16 & 0.9786106 & 0.1625290 & 0.0508156 & 0.2991672 & 0.1971504 & 0.1445413 \\
\hline 24 & 0.9786054 & 0.1625300 & 0.0508276 & 0.2991682 & 0.1971522 & 0.1445427 \\
\hline 32 & 0.9786044 & 0.1625301 & 0.0508279 & 0.2991682 & 0.1971524 & 0.1445430 \\
\hline 40 & 0.9786043 & 0.1625302 & 0.0508277 & 0.2991683 & 0.1971525 & 0.1445431 \\
\hline 60 & 0.9786041 & 0.1625302 & 0.0508276 & 0.2991684 & 0.1971526 & 0.1445431 \\
\hline 80 & 0.9786041 & 0.1625302 & 0.0508275 & 0.2991684 & 0.1971526 & 0.1445431 \\
\hline 100 & 0.9786041 & 0.1625302 & 0.0508275 & 0.2991684 & 0.1971526 & 0.1445431 \\
\hline Ref. [11] & 0.9786040 & 0.1625302 & 0.0508275 & 0.2991682 & 0.1971524 & 0.1445429 \\
\hline
\end{tabular}


TABLE V. Comparison of two-variable Nystrom calculations with and without the singularity correction term. Listed are the scattering amplitudes $A(x, E)$ at $E=0.25$ and $E=4.0 \mathrm{MeV}$. For calculations in this table, $N_{r}=144$ and $N_{x}=100$.

\begin{tabular}{|c|c|c|c|c|c|c|}
\hline \multirow[b]{2}{*}{$\hat{C}$} & \multicolumn{3}{|c|}{$\operatorname{Re} A(x ; E)$} & \multicolumn{3}{|c|}{$\operatorname{Im} A(x ; E)$} \\
\hline & $x=1.0$ & $x=0.0$ & $x=-1.0$ & $x=1.0$ & $x=0.0$ & $x=-1.0$ \\
\hline & & & $E=0.25$ & & & \\
\hline without $\hat{C}$ & 1.039150 & 0.860957 & 0.724570 & 1.500840 & 1.496759 & 1.492707 \\
\hline with $\hat{C}$ & 1.039792 & 0.861630 & 0.725286 & 1.499687 & 1.495594 & 1.491558 \\
\hline Ref. [11] & 1.039795 & 0.861633 & 0.725289 & 1.499682 & 1.495589 & 1.491553 \\
\hline & & & $E=4.0$ & & & \\
\hline without $\hat{C}$ & 0.978796 & 0.162517 & 0.050749 & 0.299362 & 0.197281 & 0.144601 \\
\hline with $\hat{C}$ & 0.978604 & 0.162530 & 0.050828 & 0.299169 & 0.197153 & 0.144544 \\
\hline Ref. [11] & 0.978604 & 0.162530 & 0.050828 & 0.299168 & 0.197152 & 0.144543 \\
\hline
\end{tabular}

\section{B. Results of two-variable calculations}

Tables III and IV report convergence of the Nystrom method for the two-variable LS equation with respect to $N_{r}$ and $N_{x}$ for the case $\mu=0.5$. Integration over $\phi$ implicit in the calculation of $\hat{G}\left(r, x ; r^{\prime}, x^{\prime}\right)$ is done with $N_{\phi}=64$, which is sufficient for the stability of results to the number of digits shown in these tables. For the most refined calculation (with $N_{r}=252$ and $N_{x}=100$ ) in Table III, the largest absolute deviation (from the reference solution) is $1.2 \times 10^{-6}$ for $E=0.25$ and $2 \times 10^{-7}$ for $E=4.0$.

Relatively high values of $N_{r}$ and $N_{x}$ were needed to achieve agreement within seven to eight significant figure. However, more moderate values such as $N_{r}=120$ and $N_{x}=60$ are sufficient to obtain agreement within five to six significant figures. We note in passing that reference results obtained from the momentum-space Nystrom method [11,13] involve about the same level of computational effort as the coordinate-space Nystrom method for comparable levels of convergence.

In order to make manifest the role that the singularity correction term $\hat{C}\left(r_{i}, x_{j}\right)$ plays in the performance of the coordinate-space Nystrom method for the reduced LS equation, Table $\mathrm{V}$ gives the results obtained by setting $\hat{C}\left(r_{i}, x_{j}\right)=0$ in Eq. (25), which corresponds to pretending as if the kernel has no singularity. As the singularity of the reduced kernel is in fact weaker, ignoring it does not lead to a catastrophe, but results are of low accuracy. Note, however, that the correction

TABLE VI. Convergence of the partial-wave expansion for the model potential with reduced mass $\mu=0.5$. Listed are the scattering amplitudes $A(x ; E)$ for different values of the maximum number $j_{\max }$ of partial waves retained in the partial-wave decomposition for three different energies.

\begin{tabular}{|c|c|c|c|c|c|c|c|}
\hline \multirow[b]{2}{*}{$E$} & \multirow[b]{2}{*}{$j_{\max }$} & \multicolumn{3}{|c|}{$\operatorname{Re} A(x ; E)$} & \multicolumn{3}{|c|}{$\operatorname{Im} A(x ; E)$} \\
\hline & & $x=1.0$ & $x=0.0$ & $x=-1.0$ & $x=1.0$ & $x=0.0$ & $x=-1.0$ \\
\hline \multirow[t]{6}{*}{0.25} & 2 & 1.038565 & 0.861600 & 0.726345 & 1.499682 & 1.495589 & 1.491554 \\
\hline & 3 & 1.039702 & 0.861600 & 0.725078 & 1.499683 & 1.495589 & 1.491553 \\
\hline & 4 & 1.039788 & 0.861633 & 0.725294 & 1.499683 & 1.495589 & 1.491553 \\
\hline & 5 & 1.039794 & 0.861633 & 0.725288 & 1.499683 & 1.495589 & 1.491553 \\
\hline & 6 & 1.039795 & 0.861633 & 0.725288 & 1.499683 & 1.495589 & 1.491553 \\
\hline & ref. soln. & 1.039795 & 0.861633 & 0.725289 & 1.499682 & 1.495589 & 1.491553 \\
\hline \multirow[t]{8}{*}{4.0} & 5 & 0.956973 & 0.165689 & 0.042653 & 0.299142 & 0.197159 & 0.144524 \\
\hline & 8 & 0.976730 & 0.162628 & 0.051569 & 0.299168 & 0.197152 & 0.144543 \\
\hline & 10 & 0.978258 & 0.162514 & 0.050967 & 0.299168 & 0.197152 & 0.144543 \\
\hline & 12 & 0.978542 & 0.162533 & 0.050853 & 0.299168 & 0.197152 & 0.144543 \\
\hline & 15 & 0.978600 & 0.162530 & 0.050826 & 0.299168 & 0.197152 & 0.144543 \\
\hline & 18 & 0.978604 & 0.162530 & 0.050828 & 0.299168 & 0.197152 & 0.144543 \\
\hline & 20 & 0.978604 & 0.162530 & 0.050828 & 0.299168 & 0.197152 & 0.144543 \\
\hline & ref. soln. & 0.978604 & 0.162530 & 0.050828 & 0.299168 & 0.197152 & 0.144543 \\
\hline \multirow[t]{8}{*}{25.0} & 10 & 0.926359 & 0.029309 & 0.024418 & 0.116918 & 0.026848 & 0.015815 \\
\hline & 20 & 0.994033 & 0.031468 & 0.014049 & 0.117073 & 0.026855 & 0.015764 \\
\hline & 30 & 0.996584 & 0.031405 & 0.013617 & 0.117073 & 0.026855 & 0.015764 \\
\hline & 40 & 0.996660 & 0.031407 & 0.013603 & 0.117073 & 0.026855 & 0.015764 \\
\hline & 50 & 0.996662 & 0.031407 & 0.013603 & 0.117073 & 0.026855 & 0.015764 \\
\hline & 60 & 0.996663 & 0.031407 & 0.013603 & 0.117073 & 0.026855 & 0.015764 \\
\hline & ref. soln. & 0.996663 & 0.031407 & 0.013603 & 0.117073 & 0.026855 & 0.015764 \\
\hline & $K$ Born & 0.975705 & 0.028311 & 0.012069 & 0.107155 & 0.022280 & 0.012565 \\
\hline
\end{tabular}


TABLE VII. Convergence of the partial-wave expansion for the model potential with reduced mass $\mu=2.0$. Listed are the scattering amplitudes $A(x ; E)$ at $E=25$ for different values of the maximum number $j_{\max }$ of partial waves retained in the partial-wave decomposition.

\begin{tabular}{|c|c|c|c|c|c|c|}
\hline \multirow[b]{2}{*}{$j_{\max }$} & \multicolumn{3}{|c|}{$\operatorname{Re} A(x ; E)$} & \multicolumn{3}{|c|}{$\operatorname{Im} A(x ; E)$} \\
\hline & $x=1.0$ & $x=0.0$ & $x=-1.0$ & $x=1.0$ & $x=0.0$ & $x=-1.0$ \\
\hline 30 & 3.705917 & -0.017113 & -0.007998 & 0.866780 & 0.038117 & 0.015948 \\
\hline 40 & 3.759423 & -0.016295 & -0.012363 & 0.866831 & 0.038118 & 0.015939 \\
\hline 50 & 3.769466 & -0.016431 & -0.013218 & 0.866833 & 0.038118 & 0.015939 \\
\hline 60 & 3.771245 & -0.016409 & -0.013373 & 0.866833 & 0.038118 & 0.015939 \\
\hline 70 & 3.771547 & -0.016413 & -0.013400 & 0.866833 & 0.038118 & 0.015939 \\
\hline 80 & 3.771597 & -0.016412 & -0.013405 & 0.866833 & 0.038118 & 0.015939 \\
\hline 90 & 3.771605 & -0.016412 & -0.013406 & 0.866833 & 0.038118 & 0.015939 \\
\hline 100 & 3.771606 & -0.016412 & -0.013406 & 0.866833 & 0.038118 & 0.015939 \\
\hline 110 & 3.771606 & -0.016412 & -0.013406 & 0.866833 & 0.038118 & 0.015939 \\
\hline ref. soln. & 3.771606 & -0.016412 & -0.013406 & 0.866833 & 0.038118 & 0.015939 \\
\hline$K$ Born & 3.688422 & -0.000702 & -0.001694 & 0.738754 & 0.021189 & 0.008837 \\
\hline
\end{tabular}

term $C\left(\mathbf{r}_{\alpha}\right)$ in (21) for the three-dimensional case plays a much more crucial role, for without it the Nystrom idea is totally inapplicable.

The results in Tables I-V indicate that the treatment of the kernel singularity via the proposed subtraction scheme and the subsequent application of the Nystrom method yield a straightforward and viable scheme for solving the LS equation in coordinate space without invoking partial-wave decomposition. In Tables VI-VIII we probe the question of how this scheme fares compared to the traditional partial-wave approach.

For the low-mass case of $\mu=0.5$ at energy $E=0.25$, for convergence within $1 \times 10^{-6}$, the partial-wave expansion requires at most five to six partial waves, whereas the Nystrom method would need 32-40 quadrature points (in the angular variable $x$ ) for similar accuracy. However, at a relatively higher energy of $E=4$, for convergence in the sixth digit after the decimal point, about 15-18 terms are needed in the partial-wave expansion as compared to about 24-32 quadrature points for $x$. At $E=25$, however, Tables VI and VIII show that the number of partial waves and the number of $x$ points needed for convergence become comparable and are on the order of 40-50. Table VI also give the scattering amplitude calculated from the Born approximation to the $K$ matrix. Even at such a high energy as $E=25$, the Born approximation is not quantitatively reliable. Tables VII and VIII also give results for the case of $\mu=2.0$. For $E=25$, the forward amplitude requires about 90 partial waves for results stable to within six digits after the decimal point. For the same level of accuracy, the present Nystrom approach requires about 100 quadrature

TABLE VIII. Convergence of the Nystrom solution of the two-variable LS equation with respect to $N_{x}$, the number of quadrature points for the variable $x$. Listed are the scattering amplitudes $A(x ; E)$ at $E=25$ with two different values of the reduced mass $\mu$ for different values of $N_{x}$. For calculations reported in this table, the number $N_{r}$ of quadrature points for the variable $r$ is 360 .

\begin{tabular}{|c|c|c|c|c|c|c|c|}
\hline \multirow[b]{2}{*}{$\mu$} & \multirow[b]{2}{*}{$N_{x}$} & \multicolumn{3}{|c|}{$\operatorname{Re} A(x ; E)$} & \multicolumn{3}{|c|}{$\operatorname{Im} A(x ; E)$} \\
\hline & & $x=1.0$ & $x=0.0$ & $x=-1.0$ & $x=1.0$ & $x=0.0$ & $x=-1.0$ \\
\hline \multirow[t]{8}{*}{0.5} & 8 & 0.997526 & 0.019851 & -0.072424 & 0.118157 & 0.026695 & 0.013368 \\
\hline & 16 & 0.996739 & 0.031101 & 0.001398 & 0.117084 & 0.026855 & 0.015748 \\
\hline & 24 & 0.996676 & 0.031401 & 0.012565 & 0.117074 & 0.026855 & 0.015764 \\
\hline & 32 & 0.996667 & 0.031405 & 0.013603 & 0.117074 & 0.026855 & 0.015764 \\
\hline & 40 & 0.996665 & 0.031407 & 0.013603 & 0.117074 & 0.026855 & 0.015764 \\
\hline & 60 & 0.996663 & 0.031407 & 0.013603 & 0.117073 & 0.026855 & 0.015764 \\
\hline & 80 & 0.996663 & 0.031407 & 0.013603 & 0.117073 & 0.026855 & 0.015764 \\
\hline & ref. soln. & 0.996663 & 0.031407 & 0.013603 & 0.117073 & 0.026855 & 0.015764 \\
\hline \multirow[t]{10}{*}{2.0} & 8 & 3.751367 & -0.085060 & -0.218222 & 0.918689 & 0.023925 & -0.047998 \\
\hline & 16 & 3.775434 & -0.034230 & -0.019319 & 0.872404 & 0.037588 & 0.005130 \\
\hline & 24 & 3.772784 & -0.019019 & -0.090612 & 0.867371 & 0.038110 & 0.014977 \\
\hline & 32 & 3.772015 & -0.018328 & -0.013429 & 0.866941 & 0.038111 & 0.015943 \\
\hline & 40 & 3.771761 & -0.016177 & -0.013406 & 0.866868 & 0.038119 & 0.015939 \\
\hline & 64 & 3.771633 & -0.016424 & -0.013406 & 0.866842 & 0.038119 & 0.015939 \\
\hline & 80 & 3.771618 & -0.016414 & -0.013406 & 0.866838 & 0.038119 & 0.015939 \\
\hline & 100 & 3.771612 & -0.016413 & -0.013407 & 0.866837 & 0.038119 & 0.015939 \\
\hline & 120 & 3.771609 & -0.016413 & -0.013406 & 0.866836 & 0.038119 & 0.015939 \\
\hline & ref. soln. & 3.771606 & -0.016412 & -0.013406 & 0.866833 & 0.038118 & 0.015939 \\
\hline
\end{tabular}


points for $x$. Again, the Born approximation does not appear reliable, especially in nonforward directions.

\section{CONCLUSION}

As part of our continuing interest in multivariable methods for solving scattering integral equations without invoking expansions over angular momentum states, we have considered the direct numerical solution of the LS integral equation in coordinate space. With the particular subtraction procedure adopted to treat the kernel singularity, the Nystrom method leads to a simple and straightforward scheme. We found that, for the model potential used in this study, the present coordinate-space approach compares favorably, in terms of its ease of implementation and computational effectiveness, with the Nystrom method for the momentum-space version of the LS equation. The coordinate-space approach is more natural for local potentials. For instance, in the context of a Faddeev-equation approach to three-atom problems [22-24], calculation of the atom-atom transition matrices (for numerically available diatomic potentials) would be more practical in coordinate space.

Both the $T$-matrix and $K$-matrix versions were implemented with excellent agreement between them. The $K$-matrix approach demands fewer computational resources because the most time-consuming task (namely, construction of the kernel matrix and solution of the resulting system of equations) is done with real arithmetic. Once real $K$-matrix elements are calculated, $T$ matrix elements are then obtained by solving the Heitler equation, which is an integral equation in angular variables only.

The choice between direct (non-partial-wave) methods and partial-wave approaches is context dependent. The natural context for the direct approach is of course two-body problems with noncentral interactions. On the other hand, for central potentials, the partial-wave approach involving the solution of a single-variable integral equation many times would appear at first sight to have an advantage over direct methods involving two or three variables. Although partial-wave approaches (in coordinate or momentum representations) involve solving $j_{\max }$ uncoupled single-variable LS equations (in radial variable $r$ or in magnitude $q$ of momentum), the kernel has to be assembled anew for each partial wave and calculation of the partialwave Green's function or the partial-wave momentum matrix elements of the potential can be taxing, especially when one has to deal with high partial waves. In contrast, the construction of the kernel matrix in the non-partial-wave coordinate-space approach is extremely simple and elementary, but the kernel dimension is $N_{x} N_{r}$. Therefore, comparisons of non-partialwave and partial-wave approaches have to take into account this difference in the organization and implementation of the respective methods. As such, the partial-wave approach is definitely advantageous at low energies for small reduced masses. However, as corroborated by our calculations, with large reduced masses and high energies, the advantages of partial-wave approach are compromised by excessively large partial waves and the vicissitudes of having to deal with Legendre polynomials and spherical Bessel functions of high order, as was experienced in fact in our partial-wave calculations for $E=25$ reported in Tables VI and VII. The recent questioning of, as well as the tendency to avoid, the partial-wave ansatz within the context of few-body problems stems from such considerations $[2,14,15]$.

The proposed method can be easily extended to two-body multichannel LS equations of the form

$$
T_{n n_{0}}=V_{n n_{0}}+\sum_{n^{\prime}} V_{n n^{\prime}} G_{0 n^{\prime}} T_{n^{\prime} n_{0}}
$$

where $G_{0 n}=\left(E-\epsilon_{n}-H_{0}\right)^{-1}$, with $\epsilon_{n}$ being the internal energy of the $n$th channel. For example, the two-channel models employed in Refs. [1,25] can be solved via a simple extension of the present method. However, the question of how one reduces a many-particle collision problem (involving a projectile incident on a target) to the matrix LS form is beyond the scope of the present contribution. We just point out that the reduction process from many-particle dynamics to effective two-body LS equations of the form of Eq. (22) often involves approximations and arguments with greatly differing levels of rigor and justification. One example of such a reduction scheme in the context of a three-particle problem with rearrangement and breakup channels has been discussed in Refs. [26,27], where effective two-body matrix LS equations for the spectator particle were obtained from Faddeev equations (and other connected-kernel three-particle equations) by approximating the two-particle resolvents in terms of two-particle bound states and pseudostates.

The present calculations show that the Nystrom method coupled with the particular singularity removal scheme adopted is a viable procedure that is capable of producing accurate solutions of the LS equation. However, matrix dimensions in the Nystrom method implemented with directproduct quadrature schemes quickly become computationally prohibitive. In fact, both coordinate- and momentum-space versions suffer from this problem. Variational methods based on multivariate bases may provide the alternative to the multivariate Nystrom method. The singularity subtraction scheme used in the present article would also be applicable in calculating the matrix elements that come up in the variational approaches and in other Galerkin-Petrov methods using basis functions in coordinate space. However, the burden of dimensionality hampers all methods that make use of direct-product bases. The radial basis function (RBF) approach (which is nearly dimension independent) has emerged in recent years as an alternative to direct-product bases $[28,29]$. In a recent article [13] we explored the use of RBFs (in momentum space) in relation to the momentum-space LS equation with promising results (for both three-dimensional and two-dimensional versions). A logical continuation of the present work would be to consider RBF expansions (in coordinate space) as a means of solving the coordinate-space LS equation and of obtaining separable expansions of the multivariable $T$ matrix. Separable expansions (of manageable rank) in multivariate bases for the two-particle $T$ matrix would be particularly useful for threebody calculations without angular momentum decomposition $[2,14,15]$.

Finally, we point out that the present coordinate-space integral-equation approach to the two-body problem might pave the way for a computational approach to three-body Faddeev equations, namely, the coordinate-space integral- 
equation version of Faddeev equations could be used as a computational vehicle, instead of the usual momentum-space integral-equation version or the integro-differential-equation version in coordinate space. Experience gained in handling the singularity of $G_{0}\left(\mathbf{r}, \mathbf{r}^{\prime}\right)$ in the two-body context could perhaps lead to similar subtraction schemes being devised for coordinate representations of two-body resolvent operators in the three-body context.
[1] A. S. Kadyrov, I. B. Abdurakhmanov, I. Bray, and A. T. Stelbovics, Phys. Rev. A 80, 022704 (2009).

[2] C. Elster, W. Glöckle, and H. Witala, Few-Body Syst. 45, 1 (2009).

[3] C. Elster, J. H. Thomas, and W. Glöckle, Few-Body Syst. 24, 55 (1998).

[4] J. Shertzer and A. Temkin, Phys. Rev. A 63, 062714 (2001).

[5] G. L. Caia, V. Pascalutsa, and L. E. Wright, Phys. Rev. C 69, 034003 (2004).

[6] B. M. Kessler, G. L. Payne, and W. N. Polyzou, Phys. Rev. C 70, 034003 (2004).

[7] A. S. Kadryov, I. Bray, A. T. Stelbovics, and B. Saha, J. Phys. B 38, 509 (2005).

[8] G. Ramalho, A. Arriaga, and M. T. Peña, Few-Body Syst. 39, 123 (2006).

[9] M. Rodríguez-Gallardo, A. Deltuva, E. Cravo, R. Crespo, and A. C. Fonseca, Phys. Rev. C 78, 034602 (2008).

[10] S. Veerasamy, C. Elster, and W. N. Polyzou, Few-Body Syst. 54, 2207 (2008).

[11] Z. C. Kuruoğlu, Few-Body Syst. 55, 69 (2014).

[12] Z. C. Kuruoğlu, J. Math. Chem. 52, 1857 (2014).

[13] Z. C. Kuruoğlu, Few-Body Syst. 55, 1167 (2014).

[14] W. Schadow, C. Elster, and W. Glöckle, Few-Body Syst. 28, 15 (2000).

[15] H. Liu, C. Elster, and W. Glöckle, Phys. Rev. C 72, 054003 (2005).
[16] G. Staszewska and D. G. Truhlar, J. Chem. Phys. 86, 2793 (1987).

[17] Y. Sun, D. J. Kouri, and D. G. Truhlar, Nucl. Phys. A 508, 41c (1990).

[18] X.-Q. Sheng and W. Song, Essentials of Computational Electromagnetics (Wiley, Singapore, 2012).

[19] M. N. O. Sadiku, Numerical Techniques in Electromagnetics (CRC, Boca Raton, 2000).

[20] K. E. Atkinson, A Survey of Numerical Methods for the Solution of Fredholm Integral Equations of the Second Kind (SIAM, Philadelphia, 1976).

[21] Z. C. Kuruoglu and D. A. Micha, J. Chem. Phys. 80, 4262 (1984).

[22] Z. C. Kuruoğlu and D. A. Micha, J. Chem. Phys. 79, 6115 (1983)

[23] D. A. Micha and Z. C. Kuruoğlu, ACS Symp. Ser. 263, 401 (1984).

[24] Z. C. Kuruoğlu and D. A. Micha, Int. J. Quantum Chem. Suppl. S23 36, 103 (1989).

[25] R. Čurík, P. Čársky, and M. Allan, J. Chem. Phys. 142, 144312 (2015).

[26] Z. C. Kuruoğlu and F. S. Levin, Ann. Phys. (NY) 163, 120 (1985).

[27] S. K. Adhikari, Z. C. Kuruoğlu, and F. S. Levin, Ann. Phys. (N.Y.) 163, 149 (1985).

[28] E. W. Cheney, Multivariate Approximation Theory: Selected Topics (SIAM, Philadelphia, 1986).

[29] W. Cheney and W. Light, A Course in Approximation Theory (AMS, Providence, 2009). 